\title{
Abortion Policy and the Economics of Fertility
}

\author{
Phillip B. Levine \\ Department of Economics \\ Wellesley College \\ And \\ National Bureau of Economic Research
}

June 2003

I would like to thank Doug Staiger for helpful conversations and Jonathan Imber for encouraging me to write this paper. The contents of this paper represent a synopsis of my book, Sex and Consequences: Abortion, Public Policy, and the Economics of Fertility, which was published by Princeton University Press. 
The debate about abortion typically involves issues of philosophy, religion, ethics, and feminism. When does life begin? Does a fetus have rights? Do women have the right to control their own reproductive functions? These issues are clearly important in determining one's position regarding the policies that regulate access to abortion. Rarely, if ever, does the debate regarding abortion policy focus on the results of economic analysis.

Yet an alternative perspective that focuses on the behavioral implications of abortion policy may yield important insights. In fact, the majority of Americans express views regarding abortion that are not directly consistent with the ideological extremes of either pro-life or prochoice. Public opinion polls routinely find that over half the population believes that abortion should be allowed under limited circumstances. If the circumstances are so important, then factors other than ideology must shape people's opinions.

President Clinton expressed a more pragmatic view of abortion policy in his address to the 1996 Democratic National Convention, stating "abortion should not only be safe and legal, it should be rare." This perspective highlights the importance of behavior over ideology. What we would prefer, according to this view, is for women to avoid unwanted pregnancies in the first place.

Recent Supreme Court decisions reinforce the importance of viewing abortion policy from a behavioral perspective. In its 1992 decision in Planned Parenthood of S.E. Pennsylvania v. Casey, the Court ruled that restrictions on abortion access would be deemed constitutional as long as they do not impose an "undue burden" on women. One way to operationalize an undue burden would be to consider how the policy affects individual behavior. For instance, if women are forced to give birth to unwanted children, one may consider this an "undue burden." 
It is within this framework that economic analysis can contribute to a discussion of abortion policy. Economics focuses on the impact of incentives on individual behavior; changes in abortion policy alter individuals' incentives regarding contraception and fertility. We can apply standard economic models to predict how we might expect individuals to respond to those changes in incentives and then apply appropriate statistical methods to test whether people actually respond in the predicted manner. We can then use these results to inform a discussion regarding abortion policy.

The purpose of this paper is to provide an overview of recent applications of economic analysis to abortion policy. Research activity related to abortion policy by economists (myself included) has intensified over the past decade or so. This literature provides theoretical insights that have been supported by empirical evidence and can usefully contribute to discussions regarding abortion policy. The vast majority of this research represents "positive" economics in that its purpose is to provide a factual characterization of the ways in which individuals actually respond to changes in abortion policy. "Normative" economics issues relate to what types of abortion policies make the most sense. Although these issues have not been the focus of economic analysis, standard economic prescriptions regarding the role of government also may be applied. This paper will largely focus on the positive elements, but I will also attempt to address the normative issues.

The main hypothesis is that abortion availability can be viewed as a form of insurance. The primary feature of insurance is its ability to protect people from downside risk. In the event of a negative outcome, any loss will be compensated. If you have car insurance, for instance, you will be protected if you have an accident or if your car is stolen. Another important feature of insurance, however, is that it may alter individual behavior if coverage is complete. If, for 
instance, you are fully reimbursed if your car is involved in an accident or if it is stolen, it may lead you to drive more recklessly or to be more likely to park in areas where there is a greater risk of car theft. These behaviors increase the likelihood of experiencing a loss.

Abortion availability has similar properties. Its primary attribute is that it provides protection from downside risk; in the event that a pregnancy results that would otherwise end in an unwanted birth, the pregnancy can be terminated. On the other hand, if abortion becomes available at a very low cost, then it may alter an individual's behavior in a way that increases the likelihood of pregnancy. These pregnancies, which likely will result in abortions, would not have occurred otherwise.

The remainder of the paper will provide a more detailed description of the theory along with an overview of the empirical evidence that can validate it. I conclude by describing the implications of this framework for policy.

\section{IMPLICATIONS FROM ECONOMIC MODELS OF ABORTION AND FERTILITY}

As in any economic model, decisions are made by weighing the relevant costs and benefits of an individual's actions. Before describing this decision-making process in more detail, it would be useful to address two preliminary considerations about the relevance of this approach to such complicated human behavior as fertility, abortion, and contraception. First, I want to clarify what I mean by the "cost" of an abortion. Often, people misconstrue the concept of economic cost to mean the monetary cost of an activity. A routine abortion costs roughly $\$ 300$ and that cost certainly needs to be incorporated into the decision-making process. Yet an abortion is physically uncomfortable and emotionally traumatic. Just going to an abortion clinic may be stressful, particularly if there are protesters outside. When I refer to the "cost" of an 
abortion, I intend to include all of these factors that make the activity unpleasant, regardless of whether they are monetary in nature. If that activity becomes more unpleasant in any dimension, monetary or otherwise, I refer to it as an increase in the cost. Importantly, abortion policy affects the cost of an abortion; the more restrictive the policy, the more costly the abortion.

Second, it is worthwhile to consider whether it is even plausible that people make decisions such as these within the framework of weighing costs and benefits. Certainly I do not mean to imply that individuals have lengthy and thoughtful discussions of the relevant costs and benefits before they decide, for instance, whether or not to engage in unprotected sexual intercourse. But there are many complicated decisions that individuals make which can be modeled in this way even if this is not exactly the physical process by which the decision is made.

For instance, drivers on undivided two-lane highways routinely decide whether they can pass the car in front of them when a car is on-coming. A physicist could model this decision based upon the speed of all of the cars involved and the distance between each of them. This model would fairly accurately be able to predict the drivers' behavior. Despite the fact that individuals are unable to instantly (if ever) solve such a complicated mathematical problem, the physicist's model is still a useful way to describe the drivers' behavior. In fact, that model can be used to make predictions about how the driver would respond to changes in conditions (i.e. how would the driver respond if the car in front accelerated). An economic model in which individuals weigh the costs and benefits before undertaking something like unprotected sexual activity would be analogous to this. Individuals are assumed to make decisions as if they are weighing the costs and benefits so that the model can be used to explain their behavior and predict how they would respond to changes in their environment. 
The specific decisions that individuals have to make are whether to abort or give birth if pregnant and how intensively to use contraception. The first decision is rather straightforward to model; it is based on a simple comparison of the relevant costs and benefits of giving birth versus having an abortion. For women who want to have a child, a birth would provide a benefit; abortion is an irrelevant option for them. For other women, however, a birth would be unwanted and impose costs. For them, they would abort if the cost of the abortion is less than the cost of having an unwanted child and give birth if the relative costs were reversed.

The decision regarding contraceptive intensity is more complicated because it is made in the presence of uncertainty. More intensive use of contraception only reduces the probability of a pregnancy, it does not eliminate it (at least until abstinence or sterilization is chosen). Therefore, with some probability a woman will become pregnant and with some probability she will not. Each outcome has an associated "payoff," where a positive payoff represents a benefit and a negative payoff is a cost. In circumstances like these when decisions need to be made in the presence of uncertainty, economists model the solution by assuming that individuals maximize the "expected payoff." Here, individuals would be assumed to choose a level of contraceptive intensity by maximizing the average of the payoffs if pregnant or not weighted by the likelihood that each outcome occurs. Since the cost of getting pregnant for women who do not want a child includes the possibility of an abortion and its associated costs, abortion costs enter into decisions regarding contraceptive intensity.

These decision making processes can be used to predict how people will respond to different levels of abortion costs. This discussion focuses on women for whom giving birth would be costly since abortion is a relevant option mainly for them. Suppose that abortion costs are very high, like they would be if, say, abortion was illegal. Contraceptive intensity would 
likely be high under these circumstances, but some pregnancies may still result. If so, some women may still choose to seek a very expensive (and potentially illegal) abortion, but many may choose to have the unwanted birth anyway.

Now consider what would happen if the cost of abortion fell. At some point, the cost of abortion would fall below the threshold of the cost of an unwanted birth. At that point, a woman who otherwise would have chosen to give birth to an unwanted child will decide to abort instead. This decline in the cost of abortion reduces the number of unwanted births. Note that if abortion costs continue to fall further, no more abortions will occur since the decision to abort the unwanted birth has already been made. Then what is the impact of a continued decline in the cost of abortion? The primary effect now will be on contraceptive intensity. As abortion becomes cheaper, the cost of getting pregnant falls as well, so there is less incentive to use more intensive methods of contraception. The result will be more pregnancies, all of which will be aborted. There will be no additional impact on unwanted births. Similarly, suppose abortion costs start out low and then rise, but remain lower than the threshold of an unwanted birth. Then contraceptive intensity will rise, pregnancies and abortions will fall, but births will not increase.

Of course, translating these theoretical predictions to the impact of changes in real world abortion policies is not straightforward. The relationship between different forms of abortion policies and the threshold of the cost of an unwanted birth is impossible to know (not to mention the fact that this threshold is likely to be different for different individuals). At this stage, the translation can only be speculative; empirical analysis is required to confirm it. If the data supports the hypotheses, then the theoretical framework just described provides a useful way to think about how people respond to abortion policy changes. 
In other work, I have hypothesized that legalizing abortion will reduce the cost of an abortion below that of an unwanted birth, leading to fewer such births. Within a legal abortion environment, however, subsequent changes in abortion access mainly affect pregnancies and abortions, not births. The most common policy changes that would fit in this category include restrictions on the use of Medicaid funds to pay for abortion, parental involvement laws, and mandatory delay laws. These policies, described below, all increase the cost of abortion for those subject to the law, so they should reduce abortions and pregnancies without increasing births, according to this view. These predictions are based upon very strong assumptions and require empirical evidence to validate them. I turn to this evidence in the next section.

\section{EVIDENCE REGARDING THE IMPACT OF ABORTION POLICY}

The main difficulty in any policy evaluation exercise is determining whether the policy itself caused the behavior. To draw a conclusion about causality, one needs to be confident that the behavior would not have taken place had the policy not occurred. This is a very high hurdle that is often difficult to surmount because it requires us to know what would have happened if the policy was not in effect (the "counterfactual"). Although the ability to draw causal conclusions is difficult, recent economic research including that regarding abortion policy has gone much further than previous work in reaching that goal.

Perhaps the strongest research design that can be used to assess causality is that of a controlled experiment. Participants are randomly divided into treatment and control groups at which point treatment group members experience some change in their environment whereas control group members go about their lives as before. The most common examples involve clinical drug trials in which the drug being tested is given to members of the treatment group 
while control group members receive a placebo. Social experiments are also conducted sometimes to test the potential impact of policy changes like reforms to the welfare system, a negative income tax, and workforce training programs. Although there are some weaknesses of controlled experiments, their strength comes from the fact that randomization results in treatment and control groups that are statistically identical. This means the control group provides a reasonable counterfactual for the treatment group. In the context of abortion policy, however, such a research design is clearly out of the question based on ethical considerations.

An alternative approach that has been successfully employed is to use quasi-experimental methods. In a quasi-experiment, a policy is implemented that happens to affect some individuals, but not others in a way that replicates the idea of creating treatment and control groups. Just like random assignment is the key to a successful controlled experiment, a strong quasi-experimental design only exists when the introduction of the policy change is unrelated to the behavior of the members of the two groups. Under this condition, if the quasi-treatment group exhibits a change in behavior relative to that of the quasi-control group, then one can interpret the policy as having a causal effect.

Changes in abortion policy often have features that enable researchers to usefully employ quasi-experimental methods. One of the best applications of these methods examines the impact of abortion legalization in the early 1970s. Although the Roe v. Wade decision legalized abortion nationwide in 1973, five states (California, New York, Washington, Alaska, and Hawaii) began allowing the procedure broadly starting in 1970. In those five states, the policy change was likely to have been quite unexpected. In California, for example, abortion became widely available after a late 1969 State Supreme Court decision invalidated an earlier law banning the procedure. In New York, abortion was legalized through new legislation that passed 
by one vote as one state senator changed his vote at the last minute. The key feature in each of these cases is that the policy change was completely unanticipated. At least the exact timing of the legalization was unrelated to any contemporaneous changes in individuals' behavior in those states. A similar argument can be made regarding the 1973 Roe v. Wade decision by the U.S. Supreme Court.

This institutional detail actually sets up a double quasi-experiment that can be used to assess the impact of abortion legalization on fertility. In 1970, women in the handful of states that legalized abortion can be thought of as a treatment group (where the quasi- prefix is dropped for ease of exposition) and women in the remainder of the country would act as a control. In 1973, the treatment reversed and the early legalizers now represent the control group while the treatment occurred for women in the remainder in the country. If abortion legalization reduced fertility, then one would expect to see a decline in births in the early legalizers relative to other states in 1971 (incorporating a lag between the abortion decision and the birth decision). The gap that emerged in the early 1970s should go away around 1974, when abortion was legal throughout the country. Evidence of that nature would provide compelling support for the notion that abortion legalization reduced fertility.

In fact, the pattern in fertility among women in the two sets of states is very close to the pattern just described. Based on this comparison across states, it appears that abortion legalization reduced births by roughly 5 percent. The impact was even greater for certain population subgroups, like nonwhites and teens where both groups experienced a 12 percent decline in births. Overall, the evidence is fairly clear that abortion legalization had a causal impact on (presumably unwanted) births, reducing them by a substantial amount. 
Since abortion was legalized in the early 1970s, changes in abortion policy have gone in the direction of imposing relatively modest restrictions in its availability. The most common forms of these restrictions include limits on the use of Medicaid funds to pay for abortions, parental involvement laws, and mandatory delay laws. In each case, similar quasi-experimental methods have been employed to estimate the effect of these policy changes on fertility outcomes. In each case, I will briefly describe the institutional history that facilitates the use of these methods before describing the evidence regarding their impact.

Medicaid is the system of health care that is provided to low-income households. In 1976, the federal government passed a law (the "Hyde Amendment") prohibiting federal Medicaid funds from being used to pay for an abortion and gave states the discretion to restrict such funding as well. The law was contested in the courts and it was not until 1980 that the U.S. Supreme Court finally ruled that such restrictions were constitutional. Virtually instantaneously 27 states instituted laws restricting the use of state Medicaid funds, so that poor women on Medicaid in these states had to pay the full monetary cost of an abortion in those states. Most of the remaining states continued to pay for these abortions.

These two groups of states effectively comprise a treatment and control group that can be used to estimate the impact of these restrictions on abortion and fertility. We can compare the pattern over time in birth and abortion rates in the treatment group states that restricted Medicaid funding to the analogous rates in control group states in which there was no policy change. If abortions fell in treatment group states relative to the control group states starting around 1981, then we can attribute this change in behavior to the policy. In fact, several studies provide support for this position; abortions did seem to fall in states restricting Medicaid funding relative to other states that did not. Moreover, this impact appears to be concentrated among lower 
income women who would be eligible for Medicaid, although this finding is weakened by data limitations.

Some of these same studies also explore the impact of Medicaid funding restrictions on birth rates. Interestingly, these studies do not find any evidence of an increase in births to accompany the decrease in abortions. The only way that these two facts can be reconciled is if pregnancies fell in response to the policy change.

The other common forms of abortion restrictions in the United States are parental involvement laws and mandatory delay laws. States that enacted parental involvement laws require minors to either notify or obtain the consent of at least one parent. Mandatory delay laws require a woman seeking an abortion to wait typically one day after registering at a clinic before obtaining an abortion. The institutional history of these two types of restrictions makes the use of quasi-experimental methods somewhat more complicated. Parental involvement laws were introduced incrementally in response to a series of Supreme Court decisions over several years which made increasingly clear that such state laws would be considered constitutional. Mandatory delay laws have trickled into place ever since the 1992 Casey decision by the Supreme Court, which ruled them to be constitutional, overturning an earlier decision that went against mandatory delay. In both cases, there is no easy way to divide the country into obvious treatment and control groups to evaluate the impact by comparing outcomes before and after the policy change.

To circumvent this problem, researchers have typically adopted alternatives that are still based upon quasi-experimental techniques. One approach is the case study. In this method, researchers choose, say, one or two states that changed their policy and then select another small number of nearby states to act as a control group. Although quasi-experimental designs like 
those described earlier can be used once these groups are formed, it is unclear whether the results are specific to the chosen states or hold more broadly. Alternatively, researchers have adopted more sophisticated econometric methods that emulate the basic features of a quasi-experimental exercise. These methods still use pre-post comparisons between treatment and control groups, but allow for the fact that the policy changes all took place at different times in different states. Without an exogenous event like a Supreme Court decision to instigate the policy change, though, one may be concerned about why states decided to change policies when they did.

Nevertheless, the collective findings from studies that examine the impact of parental involvement and mandatory delay laws are consistent with that predicted earlier. Abortions become less common when either form of restriction is imposed; the impact of parental involvement laws in this regard is concentrated on minors. But there is no evidence of an increase in births. Again, the only way to reconcile these two findings is if pregnancies decline in response to the imposition of these restrictions.

All of the evidence discussed so far has described the impact of abortion policy changes in the United States. But theories of how individuals respond to changes in abortion policy are not country-specific. We can also examine policy changes in other countries to see how fertility outcomes were affected. Although many countries have experienced changes in their abortion laws, Eastern Europe represents perhaps the best laboratory. In the late 1980s and early 1990s, a number of countries changed their abortion laws (Albania, Bulgaria, Romania, the Czech and Slovak Republics, Hungary, and Poland), some of which were coincidental to changes in governments as the Soviet Empire crumbled, but others were not. Since abortion policy has been virtually unrestricted in the republics of the former Soviet Union for several decades, those republics in Europe may make a suitable control group. Therefore, we can estimate the impact 
of changes in abortion policy by comparing the pattern over time in abortions and births between the Eastern European countries that changed their laws and the European republics of the former Soviet Union that did not.

Research that has pursued this strategy has found results comparable to those described earlier regarding the United States. When a country legalizes abortion, births fall. When a country introduces moderate restrictions on abortion access within a legal abortion environment, abortions fall but births do not rise, suggesting that pregnancies fall in response. It is important to note that the moderate restrictions that some Eastern European countries had in place required patients to support their request for an abortion with a valid reason, like financial difficulty, before an abortion could be performed. Within the framework of the economic model described earlier, the results from this research suggest that those types of restrictions did not appear to increase the cost of an abortion above that of an unwanted birth.

\section{IMPLICATIONS FOR POLICY}

The discussion so far has focused on the positive economics question about the impact of changes in abortion policy on abortion, birth, and pregnancy. As a discipline, economics is wellsuited to addressing such positive questions. We develop models and conduct statistical analyses, relying on the mathematical underpinnings to feel confident that we have learned something concrete from the exercise regarding individual behavior. Although methodological debates certainly erupt sometimes, most questions of a positive nature can be eventually answered as the research literature evolves.

Normative questions are a different matter. What form should abortion policy take is a much more difficult question to answer than what impact does a change in abortion policy have. 
Answers from the latter question can certainly be applied to help answer the former, but the solution to the normative question often requires methods that weigh the conflicting sentiments of individuals in society. Economists have a much more difficult time resolving such issues. In fact, the discussion that I will provide here will focus on the contribution that economics can make to this issue rather than a clear indication of its solution.

An important distinction that is relevant to make here is that between the public and private cost of abortion. In the discussion up to this point, the costs that I have described related to the abortion procedure represent private costs because they include those costs that a woman herself would face if she chooses to abort a pregnancy. The public cost of an abortion would incorporate those private costs plus any additional costs that society faces should a woman choose to abort a pregnancy. The difference between these two costs is something that economists call an externality. Externalities are present when the actions of one party have repercussions for other parties. A classic example of an externality is pollution, which imposes a cost to society above the production costs paid by a firm. Externalities can be positive as well, providing benefits to others besides those engaged in the activity. For instance, a public education system can promote innovation, advance the electoral process, and improve public health; all these benefits go beyond the private benefits (like higher wages) received by the individual.

If women were the only ones affected by having an abortion, then the implication for public policy would be clear; abortion should be provided without regulation (or subsidy). A woman choosing to abort or give birth would incorporate the entire private cost in making her decision and choose what is best for her based on those costs. If there is no externality, so that the private cost equals the public cost, then the decision that is best for the individual is also best 
for society. The only way that any sort of government involvement is warranted is if an externality is present. Otherwise, government intervention cannot improve upon that outcome.

What are the externalities associated with abortion? We could incorporate the traditional abortion debate into the framework of externalities. From the pro-life position, if abortion represents the taking of a life, then we would ascribe a large external cost to that child whose life is ended. From the pro-choice position, however, if we take away a woman's fundamental right to control her own reproductive functions, then we have also created a large negative externality. These two perspectives cannot easily be resolved and simply graft new words onto the traditional debate. Although I do not mean to dismiss their relevance, I will refrain from further discussion of them. A firm believer in either a strictly pro-life or pro-choice position probably would not find the analysis provided here convincing or applicable anyway. What I seek to do here is to focus on incorporating the behavioral effects of changes in abortion policy.

Abortion may generate other forms of externalities. Perhaps the most direct form is the medical services associated with the procedure that could be relegated to other purposes (called an "opportunity cost," which represents what has to be given up when an activity is chosen). Although this form of externality clearly exists, it is also probably reasonably small. Since the monetary cost of the procedure and the number performed are not that large relative to overall medical spending, it can be safely ignored.

It is difficult to concretely identify other forms of externalities, although they certainly may exist. One possibility is the social costs associated with unwanted births if they result from restrictive abortion policies. These costs may include things like medical expenses, expenditures on social services, the well-being of subsequent generations, and others. They presumably contribute to the public support for things like teen pregnancy prevention programs and concerns 
regarding non-marital fertility. Alternatively, one could point to negative public perceptions regarding the use of abortion as a form of contraception as a potential form of externality.

We can incorporate the potential presence of these externalities to help think about the value of different types of abortion policies. Consider, for instance, whether abortion should be legal. With no externality, there would be no justification for imposing any limits on abortion activity, let alone outlawing it. Moreover, if unwanted births represent a negative externality, then this would further support the case for legalized abortion since the evidence shows that legalizing abortion reduces (presumably unwanted) births. To support a position against legal abortion, one would need an externality sufficient in magnitude to counteract the impact on unwanted births and the private benefits that women themselves would receive that are associated with legal abortion.

Similar reasoning can be applied to the issue of whether we should adopt relatively moderate restrictions on abortion access. Again, without some form of externality present, no such restrictions should be imposed since the greater fertility control associated with unrestricted abortion access provides a private benefit to women. But when one incorporates externalities, the arguments against these sorts of abortion restrictions are not as strong as those regarding its legal status. Past research does not validate the notion that these restrictions increase unwanted births, so that externality which worked to support the legal status of abortion is not present here. Moreover, research shows that these sorts of restrictions tend to reduce abortions by reducing pregnancies; to the extent that the public favors that type of behavior, this would go in the direction of imposing the restriction. Yet strong support for moderate restrictions would still require that externality to overcome the private benefits that women face by unrestricted abortion access. 


\section{CONCLUDING REMARKS}

Economic analysis certainly is not the conventional way to address the topic of abortion policy. It is unlikely that many participants in the standard debate would consider this approach to be a productive addition to the discussion. Nevertheless, for the large number of individuals who do not take either ideological extreme, finding a new way to think about a complicated problem may be beneficial.

Based upon the available evidence, it appears that individuals do seem to respond in predictable ways to changes in abortion access. Those predictions are based upon an economic model which provides the interesting hypothesis that individuals may respond differently when abortion is legalized than they would if abortion access is altered within a legal abortion environment. Legalization itself is found to reduce the number of unwanted births. Beyond that, imposing moderate abortion restrictions do not appear to significantly increase unwanted births; abortions and pregnancies decline instead.

These findings can be used to inform a debate regarding the appropriate form of abortion policy, although it is inadequate to resolve that debate. The reduction in unwanted births associated with legal abortion can help justify maintaining its legality because society benefits from this reduction beyond those benefits experienced by women. On the other hand, moderate abortion restrictions do not appear to lead to additional unwanted births, so this argument cannot be used effectively to oppose them. Moreover, society may prefer to introduce policies that reduce that use of abortion as a form of contraception. But this still would need to be weighed against the value of greater abortion access that women would experience directly. Unfortunately, resolving this accounting problem is beyond the scope of economic analysis. 